\title{
Wigner-Mott insulator-to-insulator transition at pressure in charge-ordered $\mathrm{Fe}_{2} \mathrm{OBO}_{3}$
}

\author{
G. Diguet, ${ }^{1}$ G. R. Hearne,,${ }^{1, *}$ W. N. Sibanda, ${ }^{1}$ E. Carleschi, ${ }^{1}$ P. Musyimi, ${ }^{1}$ V. Pischedda,${ }^{2}$ and J. P. Attfield ${ }^{3}$ \\ ${ }^{1}$ Department of Physics, University of Johannesburg, PO Box 524, Auckland Park, 2006, Johannesburg, South Africa \\ ${ }^{2}$ Institut Lumière Matière, UMR5306 Université Lyon 1-CNRS, Université de Lyon 69622 Villeurbanne, Cedex, France \\ ${ }^{3}$ Centre for Science at Extreme Conditions and School of Chemistry, University of Edinburgh, Erskine Williamson Building, \\ King's Buildings, Mayfield Road, Edinburgh EH9 3JZ, United Kingdom
}

(Received 21 October 2013; revised manuscript received 2 January 2014; published 21 January 2014)

\begin{abstract}
Magnetic-electronic studies of mixed-valence $\mathrm{Fe}_{2} \mathrm{OBO}_{3}$ have shown that ionic charge order $(\mathrm{CO})$ is disrupted at $\sim 16 \mathrm{GPa}$. The pertinent minority-spin carrier exhibits persistent intersite electron exchange $\mathrm{Fe}^{2+} \Leftrightarrow \mathrm{Fe}^{3+}$ to well beyond this pressure. Temperature-dependent electrical transport measurements over an extended pressure range presented here demonstrate that the electronic structure remains gapped to well beyond $16 \mathrm{GPa}$. Extrapolation of data to higher pressure suggests that metallization will only prevail at $P>50 \mathrm{GPa}$. Both the persistent gapped electronic state across the $\mathrm{CO}$ instability and signature of carrier confinement to Fe-Fe dimers in the $\mathrm{Fe}^{2+} \Leftrightarrow \mathrm{Fe}^{3+}$ electron exchange are rationalized as crossover from a Wigner crystal (site centered) insulator to a dimer Mott (bond centered type) insulator- "Wigner-Mott transition" at $\sim 16 \mathrm{GPa}$. The dimer insulating state is a consequence of modulation of the relevant hopping parameter $t$ in quasi-low-dimensional features in the structure (ribbons and chains). Complementary structural studies suggest that the $a$ axis is appreciably more compressible than other crystallographic directions of the original monoclinic unit cell. Therefore, such a modulation in $t$ may arise from Peierls type distortions along the $a$ axis or else stems from intrinsic modulation in the $c$ axis direction of the unit cell. This is aided by a monoclinic $\left(P 2_{1} / c\right) \rightarrow$ orthorhombic $(P m c n)$ structural adjustment that is concurrent across the electronic transition. Pressure tuning of relative values of on-site $U / t$ and intersite $V / t$ Coulomb interaction parameters of the quasi-low-dimensional features evolve the system from site-centered to dimer-centered electron localization.
\end{abstract}

DOI: 10.1103/PhysRevB.89.035132

PACS number(s): 84.37.+q, 71.28.+d, 62.50.-p

\section{INTRODUCTION}

Charge order (CO) states may occur in strongly correlated electron systems to minimize the repulsive energy between valence electrons at the expense of their kinetic energy, leading to mixed-valence superstructures [1]. Valence electrons are then almost localized on the atomic sites due to mutual repulsions. This condensation into an electron lattice, against a background of positive ion cores, is the so-called Wigner crystallization [2].

The prime example of $\mathrm{CO}$ studied for decades is the cubic spinel $\mathrm{Fe}_{3} \mathrm{O}_{4}$-magnetite or lodestone. Condensation of carriers to form an electron lattice, i.e. $\mathrm{CO}$, occurs below the Verwey transition $T_{V} \sim 120 \mathrm{~K}$. At $T<T_{V}$, a superstructure of $\mathrm{Fe}^{(3-\delta)+}$ and $\mathrm{Fe}^{(2+\delta)+}$ mixed valence states occur $(\delta=0$ for the case of fully ionic $\mathrm{CO}$, increasing to $\delta=0.5$ for no $\mathrm{CO}$ ). The value of $\delta$ at $T<T_{V}$ is still a matter of controversy and may be of the order of $0.2-0.4[3,4]$.

The iron oxoborate $\mathrm{Fe}_{2} \mathrm{OBO}_{3}$ invoked interest some years ago due to its close relation to magnetite $[5,6]$. If tetrahedral $\mathrm{Fe}^{3+}$ in $\mathrm{Fe}_{3} \mathrm{O}_{4}$ is selectively replaced by boron, the warwickite-structured $\mathrm{Fe}_{2} \mathrm{OBO}_{3}$ arises. Nearly integer iron valence separation into $\mathrm{Fe}^{2+}$ and $\mathrm{Fe}^{3+}$ occurs, as confirmed by Mössbauer effect spectroscopy, structural refinement, and electronic structure calculations [7-9], whereas the total charge disproportionation is small, of the order of 0.1 electron per site, in the majority of CO systems $[1,10]$.

The $\mathrm{LDA}+U$ electronic-structure calculations for $\mathrm{Fe}_{2} \mathrm{OBO}_{3}$ showed that electron-electron correlations in the $3 d$

*Corresponding author: grhearne@uj.ac.za shell of Fe play a significant role [8]. An insulating CO solution only appears when a strong on-site repulsion $U$ of $\sim 5.5 \mathrm{eV}$ is included. The corresponding calculated energy gap in the electronic band structure amounts to $\sim 0.4 \mathrm{eV}$, considered to be in reasonable agreement with the activation energies obtained from electrical transport experiments [5,11]. In the absence of $U$, a metallic solution exists without $\mathrm{CO}$, at variance with experiments [5,7,11]. The difference of $t_{2 g}$-orbital occupancies for $\mathrm{Fe}^{3+}$ and $\mathrm{Fe}^{2+}$ cations in $\mathrm{Fe}_{2} \mathrm{OBO}_{3}$ amounts to $80 \%$ of the ideal ionic $\mathrm{CO}$ model. This is considered the clearest case of (ionic) $\mathrm{CO}$ and is thus archetypal in that respect [7,8]. Moreover, this is primarily driven by the interplay of strong electron correlations manifested in the relatively large Hubbard $U$, in relation to the intersite Coulomb repulsion $V$.

Applied pressure modifies interatomic distances and accordingly tunes $U / t$ and $V / t$ electronic interaction parameters, where $t$ is a pertinent transfer integral (hopping parameter determining $3 d$ bandwidth) in an appropriately constructed Hamiltonian for the system. Quasi-low-dimensional structural features of $\mathrm{Fe}_{2} \mathrm{OBO}_{3}[5,12]$ and pressure tunability of shortrange $U / t$ and longer-range $V / t$ are expected to stabilize interesting ground states [13]. This is also seen in quasi-lowdimensional organic systems investigated intensively in the last decade; for a review, see Ref. [14]. These involve regions of combinations of temperature $T, U / t$, and $V / t$ phase planes, where $\mathrm{CO}$ insulator (COI, site ordered), $\mathrm{CO}$ fluctuations, dimer Mott insulator (DMI, bond-order type), metallization, and perhaps even superconductivity may occur [15-17].

Our recent magnetic-electronic study of $\mathrm{Fe}_{2} \mathrm{OBO}_{3}$ over an extended range up to $\sim 30 \mathrm{GPa}$ directly probed the Fe valence and demonstrated severe disruption to $\mathrm{CO}$ at $\sim 16 \mathrm{GPa}$ [18]. Electron exchange $\mathrm{Fe}^{2+} \Leftrightarrow \mathrm{Fe}^{3+}$, manifested as intermediate 

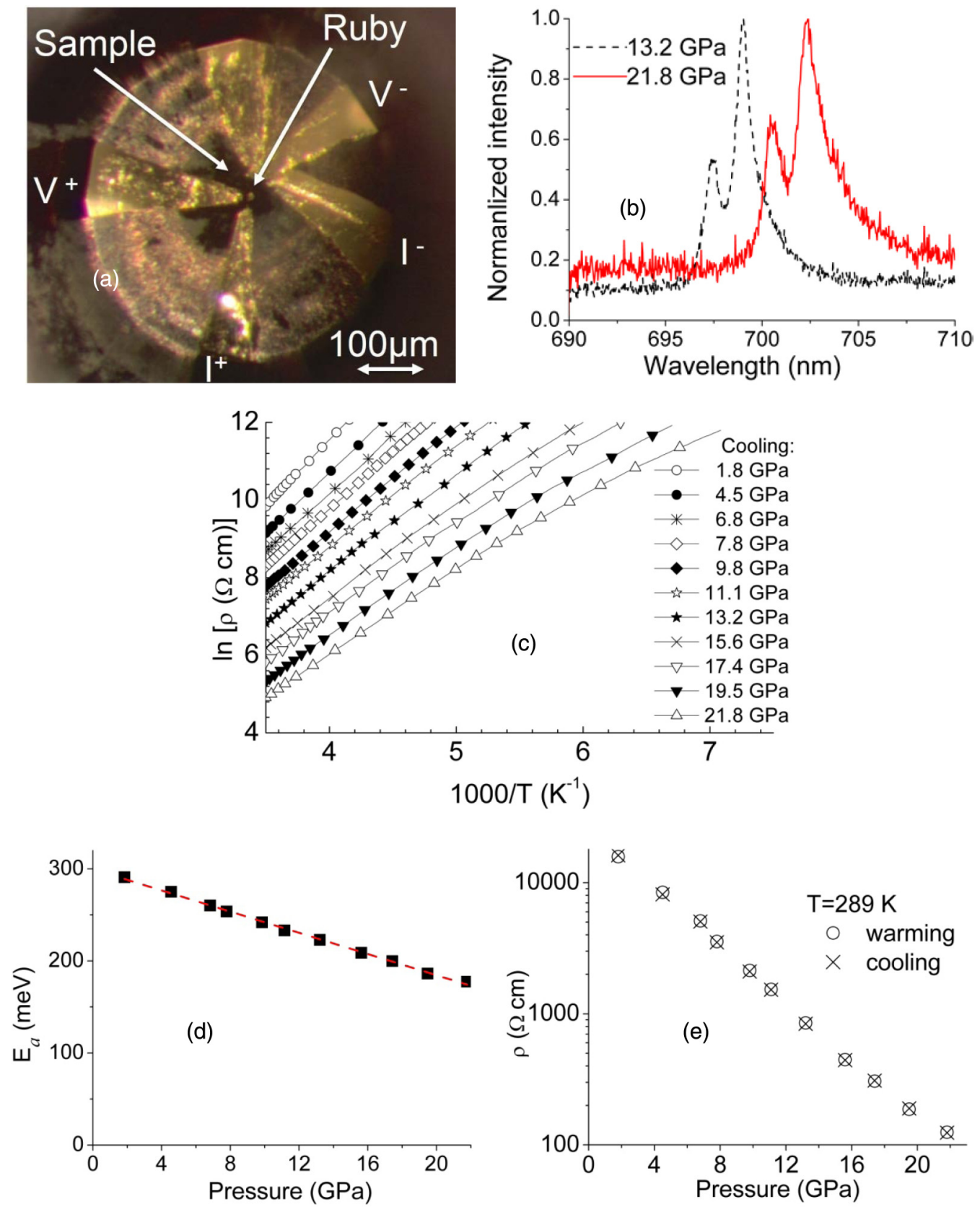

FIG. 1. (Color online) (a) Gold electrodes in a four-probe configuration within the pressurized sample cavity. (b) Examples of ruby fluorescence spectra at two different high pressures are plotted. This serves to illustrate that the fluorescence lines are well resolved to the highest pressure of this study, indicative of quasihydrostatic behavior. (c) Arrhenius plots, $\ln (\rho)$ versus $1000 / T$ at different pressures. Solid lines are to guide the eye. (d) Activation energy $E_{a}$ as a function of pressure extracted from the Arrhenius plots. (e) Plot of the resistivity at room temperature versus pressure.

valence signatures, is evidenced to well beyond this regime up to $\sim 30 \mathrm{GPa}$. To gain better insight into the nature of the disruption of this Wigner-crystal type lattice, we have investigated the pressure response of both the electrical transport and $\mathrm{x}$-ray diffraction (XRD) structural aspects to well beyond the CO instability (i.e. 16-30 GPa).

\section{EXPERIMENTAL DETAILS AND RESULTS}

Electrical-transport measurements at pressure have been performed using a diamond-anvil cell (DAC). Gold electrodes for four-probe measurements were channeled into the insulated microscopic sample cavity of the metallic gasket, see Fig. 1(a) [19]. Powdered sample from the same batch of the previous ${ }^{57} \mathrm{Fe}$ Mössbauer effect (ME) study was loaded into the insulated gasket hole [6,18]. A ruby ball for pressure determination was embedded in the sample powder, between the voltage leads [20].

Temperature-dependent resistance $(R-T)$ data to $\sim 22 \mathrm{GPa}$ has been obtained by cycling to $\sim 80 \mathrm{~K}$ in a home-developed electrical-transport station customized for the DAC, with a Si-diode in close proximity to determine temperature of the 
sample. These are converted to estimates of resistivity $(\rho-T)$ from sample and measurement geometry in Fig. 1(a). Cooling and heating curves were obtained at sufficiently low rates of $0.1-0.3 \mathrm{~K}$ minute $^{-1}$ and showed no temperature hysteresis. Pressure was determined at room temperature from the change in wavelength $\Delta \lambda(\mathrm{nm})$ of the ruby R1 fluorescence line [21]. R1 and R2 fluorescence lines could still be well resolved to the highest pressures ( $\sim 22 \mathrm{GPa})$ of the study, see Fig. 1(b), as an indicator of acceptable levels of hydrostaticity maintained in regions between the voltage leads.

The resistance-temperature behavior of $\mathrm{Fe}_{2} \mathrm{OBO}_{3}$ is that of a semiconductor (negative temperature coefficient) throughout the pressure range, 2-22 GPa, of this study. The semiconducting "gapped" behavior is best described by the following Arrhenius-type formulation for the resistivity, see Fig. 1(c):

$$
\rho=\rho_{0} e^{\frac{E_{a}}{k_{B} T}}
$$

where $E_{a}$ is the activation energy.

Here, $E_{a}$ values for each pressure, depicted in Fig. 1(d), were derived from Arrhenius plots involving Eq. (1) in the linear regime below $260 \mathrm{~K}$. A value of $E_{a}=300 \mathrm{meV}$ is extrapolated as the activation energy at ambient pressure, in good agreement with literature values [5,11]. The evolution of $E_{a}(P)$ shown in Fig. 1(d), yields a pressure dependence of $\sim 5.8 \mathrm{meV} \mathrm{GPa}^{-1}$. Metallization is then estimated to occur at 50-60 GPa, assuming the activation energy would decrease continuously to zero following a linear trend. A similar inference can be drawn from the behavior of the resistivity estimate at room temperature versus pressure, Fig. 1(e). The sample resistivity is lowered by one decade per $\sim 8 \mathrm{GPa}$. Therefore, for the resistivity to reach $1 \mathrm{~m} \Omega \mathrm{cm}$ typical of metallic conduction in strongly correlated oxides ("bad metal") [22] would still require a drop of about 5 decades beyond 22 $\mathrm{GPa}$. If there is no discontinuous electronic change in this high-pressure regime, typical metallic behavior $(d \rho / d T>0)$ would then occur at $\sim 60 \mathrm{GPa}$ or beyond [23]. Evidently strong electron correlations $(U / t \gg 1)$ and associated insulating behavior persist to extreme conditions, likely as high as 50-60 GPa. Prior ${ }^{57} \mathrm{Fe} \mathrm{ME}$ studies have shown drastic disruption to the $\mathrm{CO}$ at $\sim 16 \mathrm{GPa}$ [18], so electrical-transport measurements suggest that this $\mathrm{CO}$ instability is not associated with metallization.

The temperature pressure $(T-P)$ regime at cryogenic temperatures below $16 \mathrm{GPa}$ involves a Wigner electronic lattice ( site centered $\mathrm{Fe}^{2+}$ minority-spin carrier localization, $\mathrm{COI}$ ). At $\sim 16 \mathrm{GPa}$ and well beyond this, our previous ${ }^{57} \mathrm{Fe} \mathrm{ME}$ study demonstrated that $\mathrm{Fe}^{2+} \Leftrightarrow \mathrm{Fe}^{3+}$ electron exchange (on a time scale of $\sim 50 \mathrm{~ns}$ ) prevails at all Fe sites. This is manifested in the spectral resonance profile and its centroid (isomer shift), which lies between the values typical for "ionic" $\mathrm{Fe}^{2+}$ and $\mathrm{Fe}^{3+}$ for $P>16 \mathrm{GPa}$ [18]. Such hopping behavior, $\mathrm{Fe}^{2+} \Leftrightarrow$ $\mathrm{Fe}^{3+}$, implies that the $\mathrm{Fe}^{2+}$ minority spin carriers are confined to Fe-Fe pairs (i.e. dimers) at high pressure. The accompanying insulating behavior that ensues, $P>16 \mathrm{GPa}$ in Fig. 1, is different in nature to the site-centered localization at low pressure.

We have also investigated the structural response of the compound under pressure in XRD studies, to gain some insight into the nature of this insulating phase at high pressure [24]. Figure 2 shows selected XRD patterns of the structure at low (a)

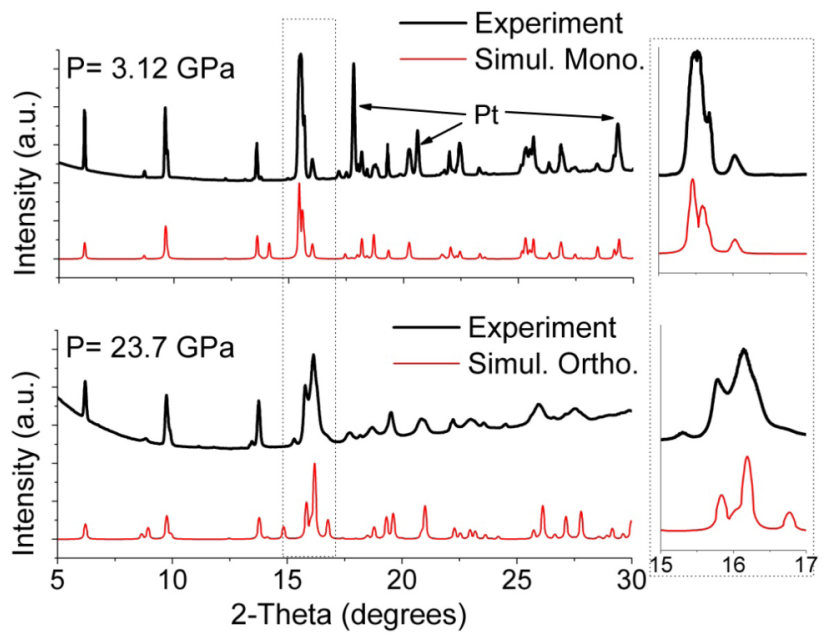

(b)

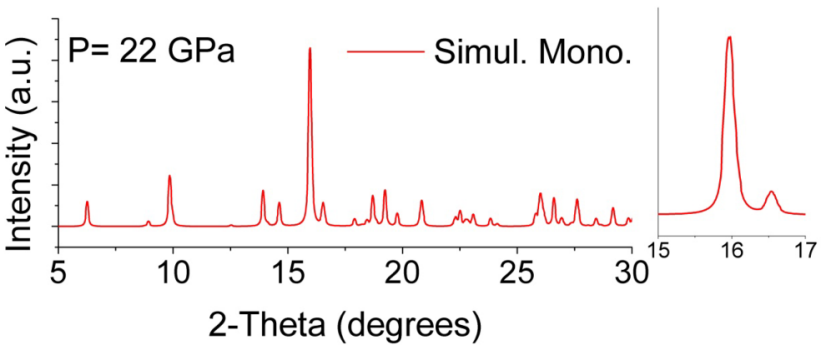

FIG. 2. (Color online) (a) Examples of x-ray diffraction patterns (black lines) taken from experiments at low and high pressures. These are accompanied by simulations (red lines) depicted below the experimental patterns to evidence the transition from a low-pressure monoclinic $\left(P 2_{1} / c\right)$ to a high-pressure orthorhombic $(P m c n)$ structural phase. Such simulations were obtained using lattice parameters derived from Rietveld refinements of the data (see text). Fingerprints of these phases are evident at the Bragg reflections near $\sim 16^{\circ}$, as shown in the expanded views in the right-hand columns. Reflections from the Pt pressure marker are shown in the spectrum at $3.12 \mathrm{GPa}$. (b) Simulation of the monoclinic phase pattern, were it to occur, as the dominant phase at high pressure, based on lattice parameters extrapolated from analysis of data at $P<16 \mathrm{GPa}$ (see also Fig. 3). The reflections at $\sim 16^{\circ}$ remain merged as a single intense feature irrespective of monoclinic angle used, unlike what is obtained in the experimental data at high pressure in (a).

pressure and at high pressure $P>16 \mathrm{GPa}$. This also has simulations of patterns for both the known (low-temperature COI) monoclinic phase $\left(P 2_{1} / c\right)$ and high-temperature orthorhombic phase (Pmcn) [7], using lattice parameters obtained from a Rietveld refinement of the XRD patterns at pressure [25]. These simulations as well as the nature of the splitting of the Bragg peaks at $16^{\circ}$ (magnified in the right-hand panels of Fig. 2) are meant to exemplify that the high-pressure phase is also orthorhombic.

The Rietveld analysis of the XRD data indicates that a progressive monoclinic to orthorhombic phase conversion occurs spanning 6-22 GPa, see Fig. 3(a). The difference in unit cell volumes for the two coexisting phases in the structural 

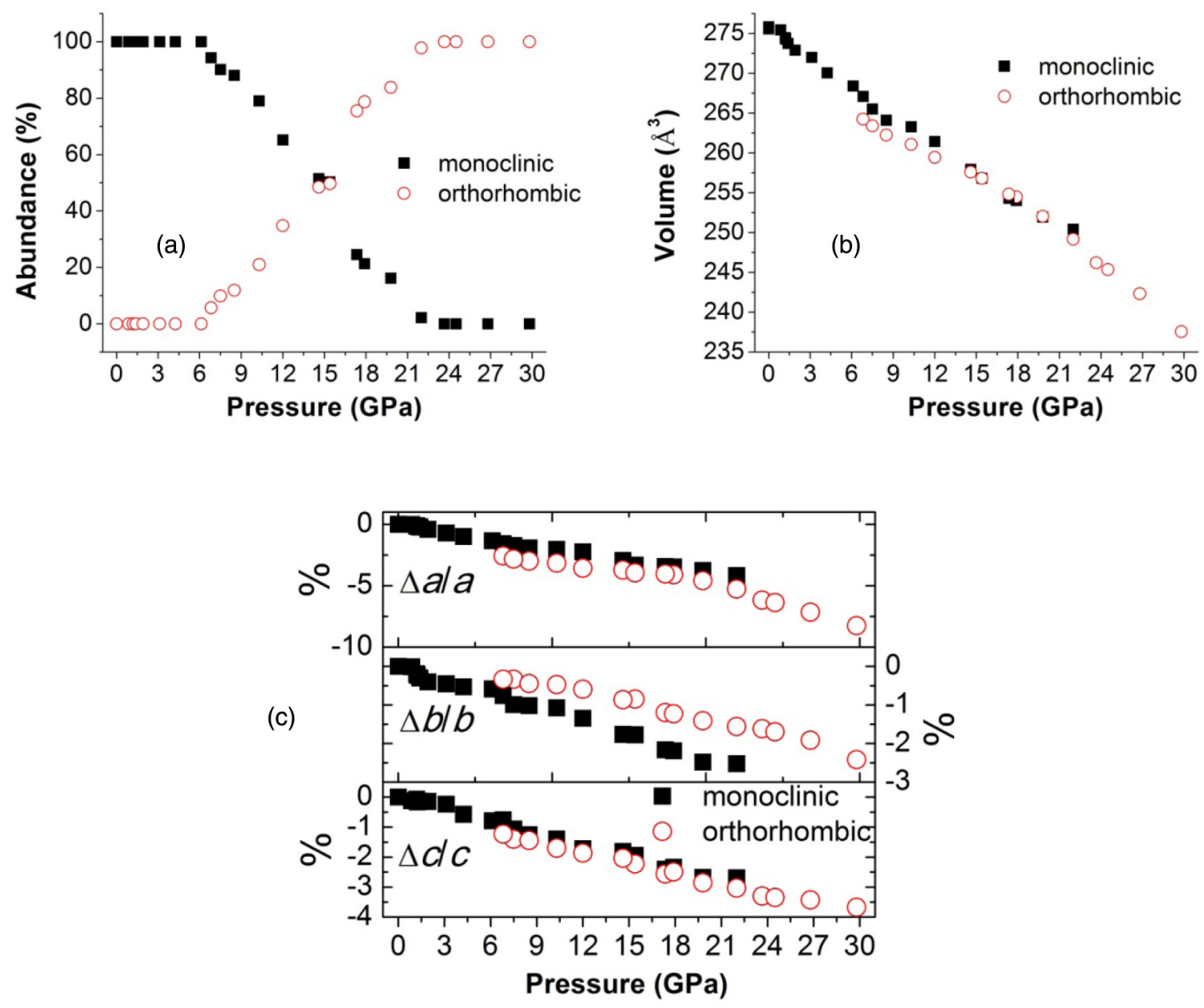

FIG. 3. (Color online) (a) Abundances of the monoclinic and orthorhombic structural phases as a function of pressure. The structural phases coexist in equal abundance at the $\mathrm{CO}$ destabilization pressure of $\sim 16 \mathrm{GPa}$ where all $\mathrm{Fe}$ sites exhibit electron exchange, $\mathrm{Fe}^{2+} \Leftrightarrow \mathrm{Fe}^{3+}$. (b) Unit cell volume of the monoclinic phase and high-pressure orthorhombic phase as a function of pressure. (c) Relative change of the lattice parameters as a function of pressure, with respect to the values at ambient pressure having $a=3.177 \AA$ as the shortest axis of the unit cell, $b=9.387 \AA$, and $c=9.251 \AA$.

evolution is small $(<1 \%)$, Fig. 3(b). The resistivity data and gap values in Fig. 1 show no discontinuous change or change in pressure dependence at the $\mathrm{CO}$ destabilization at $\sim 16 \mathrm{GPa}$. This is likely because the structural transition does not involve a significant change in either unit cell volume or its pressure dependence as well, see Fig. 3(b), to within the accuracy of the fitted lattice parameters. Furthermore, the transition is somewhat sluggish and monoclinic and orthorhombic phases occur in equal abundances at $\sim 16 \mathrm{GPa}$ in the structural conversion process that extends to beyond this electronic transition boundary. This may smear out any small change in resistivity or its pressure dependence if this occurs at the $\mathrm{CO}$ destabilization at $16 \mathrm{GPa}$. Resistivity-pressure measurements on singlecrystals would be desirable to investigate this pressure regime in more detail, especially if changes in electrical-transport at the $\mathrm{CO}$ transition are anisotropic. However, having such samples to remain intact in the pressurized cavity to such extreme conditions would pose quite a severe experimental challenge, in addition to establishing ohmic contacts on such semiconducting microscopic fragments for pressurization in a hydrostatic pressure transmitting medium. Nevertheless, the pertinent outcome of our measurements, albeit on powdered samples, is that the $\mathrm{Fe}_{2} \mathrm{OBO}_{3}$ system remains nonmetallic across the $\mathrm{CO}$ transition at $\sim 16 \mathrm{GPa}$.

The high-pressure state beyond $\sim 16 \mathrm{GPa}$ is unlikely to be a new band insulator as a result of a drastic structural modification, based on the structural response depicted in Fig. 3(b), where the difference in unit cell volume of coexisting structural phases is less than $1 \%$. The previous ${ }^{57} \mathrm{Fe}$ ME study [18] demonstrated that, beyond $16 \mathrm{GPa}$, distinct Fe valences are not discerned, and instead, a fluctuating or intermediate valence "Fe ${ }^{2.5+}$ " state occurs. This discounts a scenario of a strongly correlated phase involving site-centered charge localization in the high-pressure regime due to a strong on-site $U$, e.g. either a new mixed-valence COI state or single-valence Mott-Hubbard/charge-transfer antiferromagnetic insulator.

\section{DISCUSSION}

The combined observations that: (i) $\mathrm{Fe}_{2} \mathrm{OBO}_{3}$ is constituted by quasi-low-dimensional structural units, (ii) the high-pressure state involves electron hopping $\mathrm{Fe}^{2+} \Leftrightarrow \mathrm{Fe}^{3+}$ manifested as intermediate valence behavior, (iii) this state is nonmetallic as ascertained from the data in Fig. 1, and (iv) 
(a)

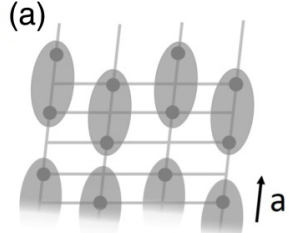

$\begin{array}{llll}2 & 1 & 1 & 2\end{array}$

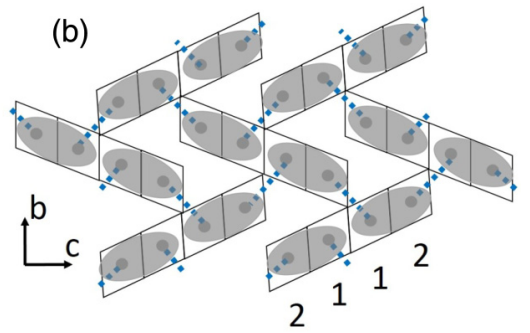

FIG. 4. (Color online) Schematic of quasi-low-dimensional aspects of the crystal structure (ribbons and chains). Crystallographic directions in the monoclinic unit cell have also been indicated. (a) Ribbon comprising four chains, extending infinitely along the $a$ axis, with distinct $\mathrm{Fe}$ octahedral sites occurring in the outer chains $\mathrm{Fe}(2)$ and inner chains $\mathrm{Fe}(1)$. (b) The $b$ - $c$ plane showing stacked ribbons. Possibilities for $\mathrm{Fe}^{2+} \Leftrightarrow \mathrm{Fe}^{3+}$ electron exchange and associated charge carrier confinement to dimers (shaded ellipses) have been depicted. These would be enhanced in the orthorhombic phase (i.e. at high temperature or high pressure).

the structural response is as depicted in Fig. 3, suggest that a somewhat unusual insulating high-pressure state occurs.

In the following, this high-pressure state is rationalized as possibly being a dimer Mott insulator involving bond-centered-type localization. This has close similarity to what has been predicted and observed in some quasi-lowdimensional systems; mainly organic conductors and in some cases transition-metal compounds [14].

At ambient pressure, $\mathrm{Fe}_{2} \mathrm{OBO}_{3}$ has a monoclinic unit cell in the COI state, comprising quasi-low-dimensional ribbons four chains wide running infinitely along the $a$ axis, see Fig. 4 . Ribbons stack laterally throughout the $b$-c plane $[8,12]$. The starting point for the theoretical description of such a COI in an array of low-dimensional (1D) lattice sites (chain) is given by the extended Hubbard model $[14,17,26]$ :

$$
\begin{aligned}
H= & -t \sum_{i} \sum_{\sigma}\left(c_{i, \sigma}^{\dagger} c_{i+1, \sigma}+\text { H.c. }\right) \\
& +U \sum_{i} n_{i, \uparrow} n_{i, \downarrow}+V \sum_{i} n_{i} n_{i+1},
\end{aligned}
$$

where $t$ is the transfer integral between neighboring sites (related to pertinent $3 d$ bandwidth). Here, $c_{i, \sigma}^{\dagger}\left(c_{i, \sigma}\right)$ creates (eliminates) an electron of spin $\sigma$ at the $i$ th site along the chain, $n_{i, \sigma}=c_{i, \sigma}^{\dagger} c_{i, \sigma}$ and $n_{i}=n_{i, \uparrow}+n_{i, \downarrow}$. Besides the kinetic energy (first) term, it includes the on-site repulsion characterized by $U$ and the nearest-neighbor Coulomb repulsion, parameterized by $V$. A strong on-site interaction $U$ favors charge disproportionation (mixed-valence states). To account for long-range $\mathrm{CO}$, intersite Coulomb repulsion $V$ must be considered. In $\mathrm{Fe}_{2} \mathrm{OBO}_{3}, U$ is of the order of 5-6 eV [8], $V \propto 1 / r_{i, i+1}$ (intersite distance) is a fraction of $1 \mathrm{eV}$ but may be up to $50 \%$ of $U$ in some compounds, and $t$ is typically a fraction of $1 \mathrm{eV}$ [14]. The extended Hubbard Hamiltonian Eq. (2) applied to highly anisotropic cases of $t$ (quasi-one-dimensionality) has been used to obtain the ground-state properties and construct phase diagrams for these systems, e.g. in the $U-V$ plane. Charge order insulator behavior occurs at comparatively large $U / t$ and $V / t$ values at $T=0 \mathrm{~K}$ and $\mathrm{CO}$ fluctuations occur at finite $T$. As these Coulomb repulsion ratios decrease (e.g.
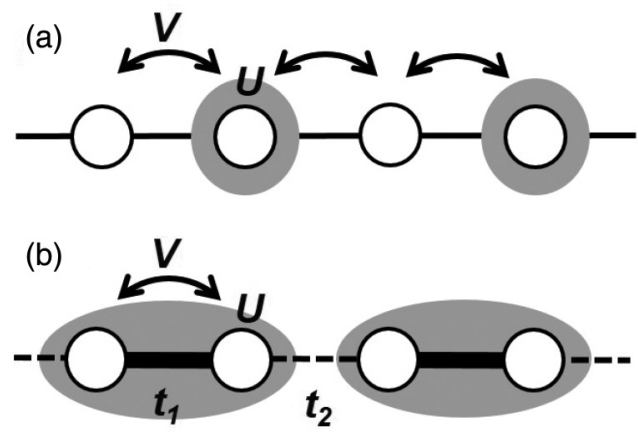

FIG. 5. Depiction of (a) CO insulator (Wigner electron lattice) and in (b) the DM insulator arising from dimerization, in a quasi-onedimensional system as adapted from Ref. [14]. Shaded areas indicate regions of valence electron localization. Symbols $V$ and $U$ refer to intersite and on-site Coulomb repulsion, respectively. Thickness of the connecting line between sites signify magnitude of the charge transfer integral $t$.

under pressure), a phase transition occurs to a metallic phase (Tomonaga-Luttinger liquid, TLL) in low dimensions (chains).

If interchain coupling is introduced into Eq. (2) [14,17]:

$$
H_{\perp}^{<j, k>}=V_{\perp} \sum_{i} n_{i, j} n_{i, k},
$$

where the summation is over pairs of adjacent sites on chains $\langle j, k\rangle$ and the strength of the interchain Coulomb repulsion is parameterized by $V_{\perp}$, then $\mathrm{CO}$ prevails at finite temperatures in the $T-(V / t)$ phase diagram with appropriately large values of $U / t$ and $V / t$ [27]. Metallization is still anticipated to occur below a critical value of $V / t$, as exemplified in various quasi-low-dimensional molecular conductors [14,28]. Applied pressure affects inter- and intrachain distances, thus tuning $U / t$ and $V / t$ to the extent that metallic behavior ensues when $\mathrm{CO}$ is destabilized. This has been exemplified in organic compounds where the pressure evolution from $\mathrm{COI}$ to metallic behavior occurs, e.g. in DI-DCNQI ${ }_{2} \mathrm{Ag}$ [28].

In $\mathrm{Fe}_{2} \mathrm{OBO}_{3}$ at $\sim 16 \mathrm{GPa}$, metallization does not ensue, although $\mathrm{CO}$ is destabilized-see one of the main results of this investigation, in Fig. 1. A different insulating phase prevails to well beyond $\sim 16 \mathrm{GPa}$, in which site-centered $\mathrm{CO}$ is absent. Such insulator-to-insulator transitions have also been investigated in quasi-low-dimensional systems which evolve from an original COI state [27,29]. Charge localization may persist and change from being site centered to bondcentered type if a strongly anisotropic $t$ prevails, involving modulated hopping strengths $t_{1}$ and $t_{2}$ amongst site pairs along the chain direction where it is strongest, see Fig. 5. This "dimerization", depicted in Fig. 5(b), may be intrinsic as originated from bonding/structural anisotropy typical of quasilow-dimensional systems. Alternatively, such dimerization may be spontaneous arising from electron-lattice coupling in Peirels-type distortions, provided that the elastic energy cost does not supersede the electronic energy gain. Both such intrinsic and spontaneous dimerization are incorporated in the kinetic term of the quasi-one-dimensional extended Hubbard 
Hamiltonian as follows [17]:

$$
H=\sum_{j} H_{1 D}^{j}+\sum_{\langle j k\rangle} H_{\perp}^{\langle j k\rangle},
$$

where

$$
\begin{aligned}
& H_{1 D}^{j} \\
& =-\sum_{i} \sum_{\sigma}\left(\left[t+(-1)^{i} \delta_{D}\right]\left(1+u_{i, j}\right)\left[c_{i, j, \sigma}^{\dagger} c_{i+1, j, \sigma}+\text { H.c. }\right]\right) \\
& \quad+\frac{K_{p}}{2} \sum_{i} u_{i, j}^{2}+U \sum_{i} n_{i, j, \uparrow} n_{i, j, \downarrow}+V \sum_{i} n_{i, j} n_{i+1, j} .
\end{aligned}
$$

The modulation of $t$, as $t-\delta_{D}$ and $t+\delta_{D}$, arises from low dimensionality features and structural details or is originated from a lattice distortion $u_{i, j}$ (at site $i$ in the $j$ th chain). Here, $K_{p}$ is the elastic coupling constant, and there may be higher-order terms. The terms in Eq. (4b) represent the intrachain part. The term $H_{\perp}^{\langle j k\rangle}$ is the interchain part [Eq. (2)] leading to CO transitions at finite temperature. Sufficiently strong dimerization leads to the dimer Mott insulator (DMI) ground state instead of metallization, in appropriate regions of the $U / t-V / t$ phase plane [17,29]. In contrast to charge being localized on every alternate site (COI), rather, valence charge localizes on every pair of sites (dimers), schematized in Fig. 5. The "on-dimer" $U$ now plays the crucial role of inhibiting dimer to dimer hopping (DMI). The system evolves from the COI ground state at large $U / t$ and $V / t$ values to the DMI state upon tuning these ratios to smaller values (e.g. by pressure to increase bandwidth $t$ ). That is, an insulator-to-insulator transition involving persistent electron correlations may occur [27,29].

The ribbon and chain features of the warwickite structure and how it sustains $\mathrm{CO}$ are depicted in Fig. 4. Charge order is predicted to thread along the chains of the $a$ axis, Fig. 4(a) [8]. Electronic structure calculations reveal the most favorable charge transfer paths conducive to hopping and thus dimer formation $[8,30]$. They may be paths involving crystallographically similar sites $\left[\right.$ e.g. $\left.\mathrm{Fe}(1) \mathrm{O}_{6}-\mathrm{Fe}(1) \mathrm{O}_{6}\right]$ within chains comprising a ribbon, Fig. 4(a). Intrinsic modulation of $t$ along the $a$ axis within a chain is absent. Therefore, dimer formation along the quasi-one-dimensional chains would need to be spontaneous Peierls type involving a lattice distortion, when energetically favored in the structure by a high enough compressibility in that direction [7,31]. Alternatively, Fig. 4(b) shows inter-intra-inter-intra-... ribbon sequences in the $c$ axis direction which involve an intrinsic modulation of $t$, similar to Fig. 5(b). Both scenarios of Fig. 4 would be similarly represented in the orthorhombic phase (e.g. at high temperature at ambient pressure) where the hopping integrals are appreciably enhanced [30].

The structural response of $\mathrm{Fe}_{2} \mathrm{OBO}_{3}$ at pressure, depicted in Fig. 3, favors the dimerization portrayed in Fig. 4. The orthorhombic structure at high pressure has a local geometry more favorable to intraribbon hopping of Fig. 4 as indicated in semi-empirical electronic structure calculations [30]. Figure 3(c) shows the relative change in lattice parameters at pressure. The changes in $a$ and $c$ axes are compatible with the dimerization schemes of Fig. 4. The shorter $a$ axis of the unit cell is much more compressible than other crystallographic directions. Spontaneous Peierls-type dimerization would therefore be favored to occur along this direction.

Previous ${ }^{57} \mathrm{Fe} \mathrm{ME}$ data show an average valence of $\mathrm{Fe}^{2.5+}$ at all $\mathrm{Fe}$ sites as a result of the electron exchange [18]. This supports the notion of dimer formation in the electronexchange process $\mathrm{Fe}^{2+} \Leftrightarrow \mathrm{Fe}^{3+}$, and not larger units like trimers that occur in $\mathrm{Fe}_{3} \mathrm{O}_{4}$ [4]. These are prevalent in both structural phases which have equal abundances at $16 \mathrm{GPa}$, see Fig. 3(a).

Pressures to $30 \mathrm{GPa}$ primarily tune the modulated $t$, described in Eq. (4), such that $\mathrm{Fe}_{2} \mathrm{OBO}_{3}$ evolves from COI in the $U / t-V / t$ phase plane to where the DMI phase is stabilized [27,29]. This may be compared with $\mathrm{Fe}_{3} \mathrm{O}_{4}$ in the same pressure regime, where an insulator-metal transition ensues [32]. The contrasting pressure responses of $\mathrm{Fe}_{3} \mathrm{O}_{4}$ and $\mathrm{Fe}_{2} \mathrm{OBO}_{3}$ may partly be ascribed to the difference in starting (ambient) pressure ground states. The COI state in $\mathrm{Fe}_{3} \mathrm{O}_{4}$ involves electron localization over three-Fe-site units (a first-approximation $\mathrm{Fe}^{2+}$ site and two $\mathrm{Fe}^{3+}$ neighbors) [4] This creates "trimeron" distortions with short Fe-Fe distances and reduces the charge separation in the $\mathrm{Fe}^{(3-\delta)+}$ and $\mathrm{Fe}^{(2+\delta)+}$ superstructure to $\delta \sim 0.4[3,4]$, compared with ionic $\mathrm{CO}$ $(\delta \sim 0)$ in $\mathrm{Fe}_{2} \mathrm{OBO}_{3}[7]$.

\section{CONCLUSION}

The pressure-induced $\mathrm{CO}$ instability at $\sim 16 \mathrm{GPa}$ in $\mathrm{Fe}_{2} \mathrm{OBO}_{3}$ can be rationalized as a change from site-centered to dimer-centered (bond centered type) charge localization, $\mathrm{COI} \rightarrow$ DMI transition. Persistent insulating behavior at $P>$ $16 \mathrm{GPa}$ in tandem with evidence of $\mathrm{Fe}^{2+} \Leftrightarrow \mathrm{Fe}^{3+}$ electron exchange and associated " $\mathrm{Fe}^{2.5+}$ " intermediate valence at all sites, from a previous direct probe of the Fe electronic state using ${ }^{57} \mathrm{Fe} \mathrm{ME}$ spectroscopy, supports the notion of electron confinement to $\mathrm{Fe}-\mathrm{Fe}$ dimers and not new site-centered localization. The structural response of the system gleaned from XRD pressure studies evidences a sluggish monoclinicto-orthorhombic transition without a significant change in unit cell volume associated with the structural adjustment. These observations suggest that the insulating high-pressure phase is unlikely to be a new band insulator that would emanate from a significant change in structure, nor is it either a new COI state or antiferromagnetic Mott insulator, both of which involve site-centered localization. Rather, there are the prerequisite quasi-low-dimensional features in the structure (chains and ribbons) and attendant anisotropy and modulation in the relevant transfer integrals $t$, for stabilizing a DMI ground state when both pertinent Coulomb repulsion parameters $U / t$ and $V / t$ are varied under pressure. Modulation in $t$ arises either spontaneously in Peierls-type distortions along the much more compressible $a$ axis or else originates from intrinsic features in the $c$ axis direction. This is compatible with the structural pressure response which evidences both a monoclinic $\rightarrow$ orthorhombic transition that enhances these transfer integrals as well as a unit cell deformation response, involving a comparatively pronounced $a$ axis compressibility, that supports the proposed dimerization schemes. Furthermore, reduced intra- and interribbon spacings under applied pressure enhance interactions between the ribbons and chains $\mathrm{V}_{\perp}$, which also favor transitions between such COI/DMI ground states. 


\section{ACKNOWLEDGMENTS}

Funding for this project has been derived from both the URC-UJ and the NRF (SA), and both EPSRC and the Royal Society (UK) and is acknowledged with gratitude.
Funding from the SISTER-MAE program enabled the work at the ELETTRA synchrotron (XRD1 beamline). We also acknowledge the loan of conductivity apparatus from A. M. Strydom (UJ) and the commissioning of that measuring station by C. Kasl.
[1] J. P. Attfield, Solid State Sciences 8, 861 (2006).

[2] E. Wigner, Phys. Rev. 46, 1002 (1934).

[3] J. P. Wright, J. P. Attfield, and P. G. Radaelli, Phys. Rev. Lett. 87, 266401 (2001).

[4] M. S. Senn, J. P. Wright, and J. P. Attfield, Nature 481, 173 (2011).

[5] J. P. Attfield, A. M. T. Bell, L. M. Rodriguez-Martinez, J. M. Greneche, R. J. Cernik, J. F. Clarke, and D. A. Perkins, Nature 396, 655 (1998).

[6] J. P. Attfield, A. M. T. Bell, L. M. Rodriguez-Martinez, J. M. Greneche, R. Retoux, M. Leblanc, R. J. Cernik, J. F. Clarke, and D. A. Perkins, J. Mater. Chem. 9, 205 (1999).

[7] M. Angst, P. Khalifah, R. P. Hermann, H. J. Xiang, M.-H. Whangbo, V. Varadarajan, J. W. Brill, B. C. Sales, and D. Mandrus, Phys. Rev. Lett. 99, 086403 (2007).

[8] I. Leonov, A. N. Yaresko, V. N. Antonov, J. P. Attfield, and V. I. Anisimov, Phys. Rev. B 72, 014407 (2005).

[9] At low temperatures $T<280 \mathrm{~K}$, the electronic system is commensurately charge ordered, with its basic charge-ordered units being chains of alternating valences, $\mathrm{Fe}^{2+}$ and $\mathrm{Fe}^{3+}$, running along the $a$ axis of the monoclinic unit cell. The total charge order is established over domains. These are large in the $a$ and $b$ directions, but of small size along the $c$ axis. In a slightly higher temperature range, for $280 \mathrm{~K}<T<$ $340 \mathrm{~K}$, the incommensurate phase occurs. It arises due to easy domain wall creation, which results most likely from a compromise between the tendency to charge ordering and the geometrical frustration for interactions between chains and ribbons of the quasi-low-dimensional structure. At $T>340 \mathrm{~K}$, $\mathrm{CO}$ is disrupted.

[10] Yundi Quan, V. Pardo, and W. E. Pickett, Phys. Rev. Lett. 109, 216401 (2012).

[11] A. P. Douvalis, V. Papaefthymiou, A. Moukarika, T. Bakas, and G. Kallias, J. Phys.: Condens. Matter 12, 177 (2000).

[12] M. Angst, R. P. Hermann, W. Schweika, J.-W. Kim, P. Khalifah, H. J. Xiang, M.-H. Whangbo, D.-H. Kim, B. C. Sales, and D. Mandrus, Phys. Rev. Lett. 99, 256402 (2007).

[13] A. Akrap, M. Angst, P. Khalifah, D. Mandrus, B. C. Sales, and L. Forró, Phys. Rev. B 82, 165106 (2010).

[14] H. Seo, C. Hotta, and H. Fukuyama, Chem. Rev. 104, 5005 (2004).

[15] K. C. Ung, S. Mazumdar, and D. Toussaint, Phys. Rev. Lett. 73, 2603 (1994).

[16] H. Yoshioka, M. Tsuchiizu, and H. Seo, J. Phys. Soc. Jpn. 75, 063706 (2006).

[17] H. Yoshioka, Y. Otsuka, and H. Seo, Crystals 2, 996 (2012).

[18] G. R. Hearne, W. N. Sibanda, E. Carleschi, V. Pischedda, and J. P. Attfield, Phys. Rev. B 86, 195134 (2012).

[19] A Merill-Basset (opposing plates) DAC comprised of Inconel low thermal expansion components has been used for the variable-temperature electrical-transport measurements to elevated pressures. The metallic gasket has been insulated with a mixture of Stycast 1266 and $\mathrm{Al}_{2} \mathrm{O}_{3}$ powder of $0.3-\mu \mathrm{m}$ grain size. The sample has been confined in a $\phi \sim 100-\mu \mathrm{m}$ insulating ring within the insulated metallic gasket cavity. Flattened gold wires were used for the electrodes in the experiment in standard four-probe measurements. An excitation current of $1 \mu \mathrm{A}$ has been used, and the measured voltage was between two leads spaced $\sim 50 \mu \mathrm{m}$ apart. The leads were configured so as to be able to establish that there is continuity of the electrodes leading into the cavity (useful in the case of insulating/semiconducting samples).

[20] This permits measurement of pressure in the vicinity of where the voltage difference is detected. It also enables a check for the degree of hydrostaticity, through the resolution of the ruby R1 and R2 fluorescence peaks. No pressure-transmitting medium has been used, and the pressure is presumably transmitted through the relatively soft sample and surrounding soft insulating materials.

[21] N. Tateiwa and Y. Haga, Rev. Sci. Instrum. 80, 123901 (2009).

[22] N. E. Hussey, K. Takenaka, and H. Takagi, Phil. Mag. 84, 2847 (2004).

[23] Electrical transport measurements at higher pressure beyond $\sim 22 \mathrm{GPa}$ were not attempted because of the perceived risk of anvil failure, given the rather large diamond culet sizes used here $(\sim 550 \mu \mathrm{m})$. Moreover, from these extrapolations, it is clear that appreciably higher pressures $P>30 \mathrm{GPa}$ would be needed to render the sample metallic. It may be noted that the highest pressure attained here $\sim 22 \mathrm{GPa}$ is sufficiently well beyond where the previous ${ }^{57} \mathrm{Fe} \mathrm{ME}$ data reveals a disruption of $\mathrm{CO}$ at $\sim 16 \mathrm{GPa}$.

[24] Angle dispersive XRD measurements were performed at the ELETTRA synchrotron (Trieste-Italy). Miniature pistoncylinder DACs were used to attain high pressures for the studies at a wavelength of $0.70001 \AA$. Daphne oil was used as a pressure transmitting medium. Two separate compression runs to high pressure, 24-30 GPa, were completed at $300 \mathrm{~K}$. Pressure estimates were obtained from the equation-of-state (EOS) of a piece of platinum loaded in the cavity (150-250 $\mu \mathrm{m}$ diameter) containing the sample. We could locate the beam $(\sim 70 \mu \mathrm{m}$ diameter) in the center of the pressurized cavity to measure predominant platinum reflections and thus estimate pressure, or have the beam slightly off the center (by 30-50 $\mu \mathrm{m}$ ) of the piece of platinum to obtain the diffraction pattern dominated by sample reflections. Numerous patterns were taken in pressure increments of 2-3 GPa. Results of the detailed analysis of the data will be published elsewhere.

[25] V. Petricek, M. Dusek and L. Palatinus, (2006). Jana2006. The crystallographic computing system. Institute of Physics, Praha, Czech Republic.

[26] H. Seo and H. Fukuyama, Synthetic Metals 133-134 257 (2003). 
[27] H. Yoshioka, M. Tsuchiizu, and H. Seo, J. Phys. Soc. Jpn. 76, 103701 (2007).

[28] T. Itou, K. Kanoda, K. Murata, T. Matsumoto, K. Hiraki, and T. Takahashi, Phys. Rev. Lett. 93, 216408 (2004).

[29] M. Tsuchiizu, H. Yoshioka, and Y. Suzumura, J. Phys. Soc. Jpn 70, 460 (2001).

[30] M. Matos and R. B. Oliveira, J. Phys.: Condens. Matter 18, 8267 (2006).

[31] A lattice distortion associated with the dimerization changes the lattice periodicity. Intersite transfer integrals would be modulated in the process. Energy gaps appear at the new Brillouin zone boundaries, and energies of occupied electronic states decrease, resulting in electronic energy gain. Such a lattice distortion $u_{i}$ is favored if the electronic energy gain, $\sim\left(\ln u_{i}\right) u_{i}{ }^{2}$, exceeds the elastic energy cost to deform the lattice $\sim 1 / 2 K_{p} u_{i}{ }^{2}$, where $K_{p}$ is related to the compressibility. See D. Khomskii, Basic aspects of the quantum theory of solids: order and elementary excitations (Cambridge Univ. Press, Cambridge England, 2010).

[32] N. Môri, S. Todo, N. Takeshita, T. Mori, and Y. Akishige, Physica B 312-313, 686 (2002). 